\title{
Childhood Cerebral Hemiatrophy (Dyke-Davidoff-Masson Syndrome) Presenting with Seizures: A Report of two Cases.
}

\author{
Danfulani Mohammed ${ }^{1}$, Gele I. Haruna ${ }^{1}$, Ma'aji M. Sadisu ${ }^{1}$, Saidu A. Sule ${ }^{1}$, \\ Muhammad A. Musa ${ }^{2}$ \\ ${ }^{I}$ (Department Of Radiology, Faculty Of Clinical Sciences, College Of Health Sciences, Usmanu Danfodiyo \\ University, Sokoto, Nigeria). \\ ${ }_{2}^{2}$ (Department Of Anatomy, Faculty Of Basic Medical Sciences, College Of Health Sciences, Usmanu Danfodiyo \\ University, Sokoto, Nigeria).
}

\begin{abstract}
Childhood cerebral hemiatrophy or Dyke-Davidoff-Masson syndrome is very rare in our locality. characterized by symmetrical calcifications noticeable in parts of the brain. We present two cases of cerebral hemiatrophy (Dyke-Davidoff-Masson syndrome) in a 4 year old boy and 8 year old girl both presenting with seizures and diagnosed using computed tomography.
\end{abstract}

Keywords: childhood, cerebral hemiatrophy, seizures, computed tomography (CT)

\section{Introduction}

Childhood cerebral hemiatrophy or Dyke-Davidoff-Masson syndrome is an uncommon clinical entity [1,2]. It is characterized by hemiplegia/paresis and seizures as well as mental retardation [1] and occurs in both sexes (males and females) with a predominance of 73.5\% in males [3]. In 1933, Dyke Davidoff and Masson first described the syndrome in a series of 9 patients with hemiplegia and cranial asymmetry on plain skull radiograph [3]. The aetiology may be congenital or acquired. A diagnosis of the condition is usually made during childhood, adolescence or adulthood. Computed tomography (CT) and magnetic resonance imaging (MRI) are the preferred imaging modalities in assessment of the aetiology and extent of lesion in the atrophied cerebral parenchyma [4]. This is a report of two cases of childhood cerebral hemiatrophy who presented with seizures and the disease was diagnosed by CT.

\section{Case Report}

A 4 year old boy who presented to the paediatric outpatient department of Usmanu Danfodiyo University Teaching Hospital, Sokoto with 1 year history of recurrent febrile convulsion. The convulsion was generalized tonic-clonic and associated with post ictal sleep. No urinary or faecal incontinence. There was history of febrile illness at 1 year of age with associated loss of consciousness. No history of traumatic head injury in the past. The antenatal and perinatal periods were uneventful. There was no history of delayed milestones. He is the second child of the parents. No family history of seizure disorder.On examination, the child was conscious, not pale, anicteric. No facial asymmetry. Neurological examination was unremarkable. A clinical assessment of seizure disorder was made. The haematological and cerebrospinal fluid examinations were normal. EEG showed normal background activity for age.He was referred to the Radiology Department for cranial computed tomography (CT) which showed prominence of the cerebral sulci in the right cerebral hemisphere with dilated ipsilateral lateral ventricle (fig. 1 and 2). The left cerebral hemisphere and the cerebellum are normal. The paranasal sinuses are also normal. An assessment of right cerebral hemiatrophy was made.The patient was placed on anticonvulsant (carbamazepine) with good seizure control. He is on routine follow up.

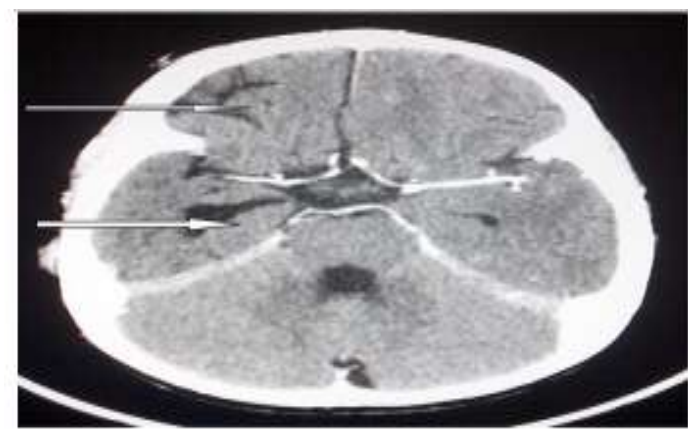

Figure 1: Contrast enhanced axial brain CT showing dilated right cerebral sulci (long arrow) and dilated temporal horn of right lateral ventricle(short arrow) 


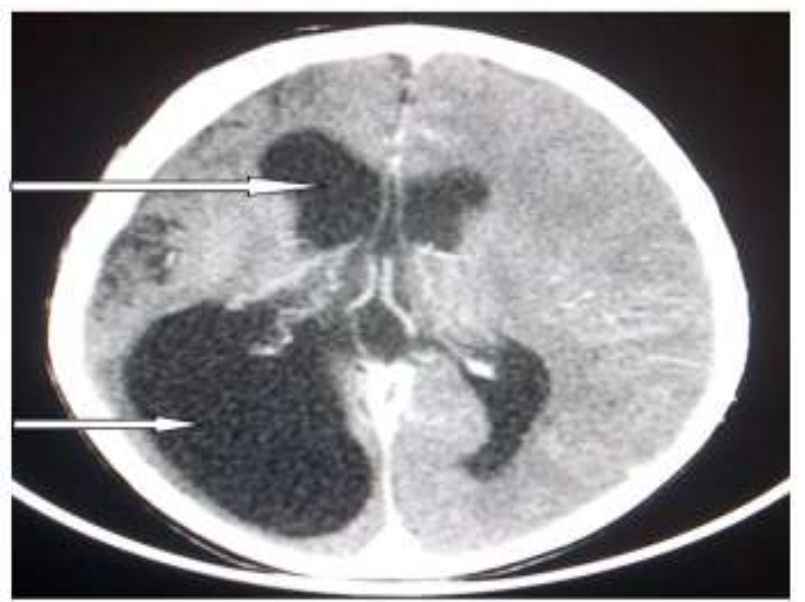

Figure 2: Contrast enhanced axial brain CT showing dilated anterior horn (long arrow) and posterior horn (short arrow) of the right lateral ventricle.

\section{Case Report}

An 8 year old girl who presented to the paediatric outpatient department of Usmanu Danfodiyo University Teaching Hospital, Sokoto with 5 years history of seizures and intellectual disability. Parents reported a previous febrile illness with repeated convulsions and prolonged coma. She was managed at home as parents ascribed the illness to spiritual cause. She lost the previously acquired motor, language and self help skills after regaining consciousness. The motor and language skill were gradually regained 4-6 months after the illness. She is the $6^{\text {th }}$ child in a monogamous setting with 10 children. No history of seizure disorder in the family.On examination she was not ill-looking, not hyperactive, not pale and anicteric. Neurological examination shows facial asymmetry and left hemiparesis. A clinical assessment of seizure disorder to rule out intracranial space occupying lesion was made and she was referred for investigations including cranial computed tomography. Laboratory examinations were unremarkable. Electroencephalography (EEG) showed intermittent bursts of generalized spikes, sharp slow waves and independent occasional bi-temporal spikes suggestive of seizure foci.Cranial CT showed prominence of the sulci involving the right cerebral hemisphere, mild dilatation of the right lateral ventricle and ipsilateral shift of the midline (Figures 3 and 4). There was right sided calvarial thickening and enlargement of the right ethmoidal sinus and right mastoid air cells (Figures 5 and 6).She was placed on anticonvulsant with good seizure control, now on routine follow up at the outpatient clinic.

Figure 3: Contrast enhanced axial brain CT showing dilated right cerebral sulci (short arrow) and dilated frontal horn of right lateral ventricle (long arrow).

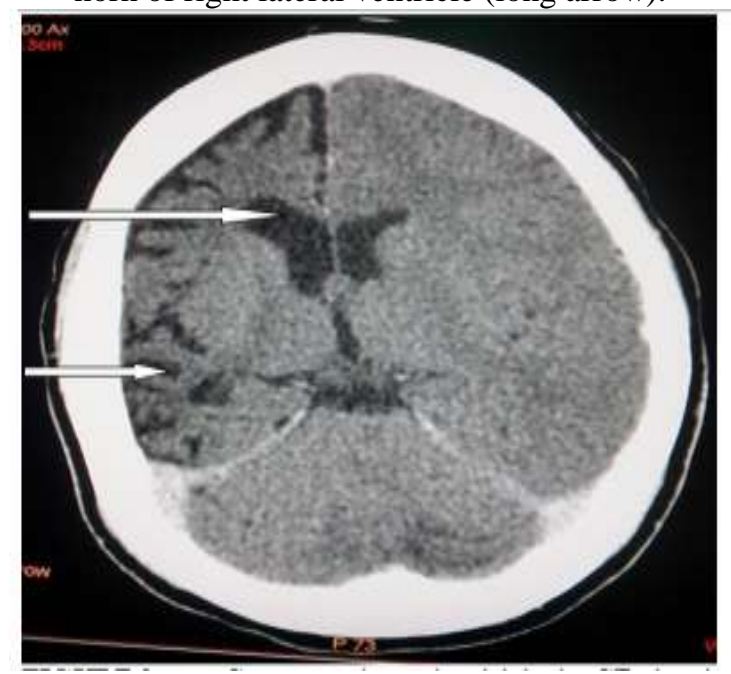




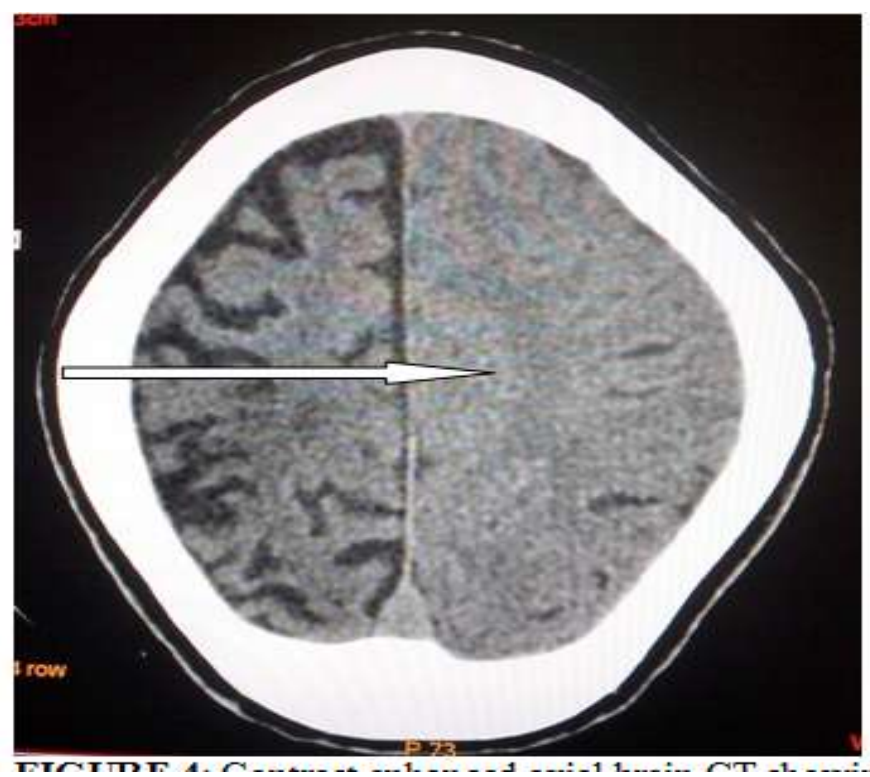

FIGURE 4: Contrast enhanced axial brain CT showing midline shift to the right due to hemiatrophy (arrow).

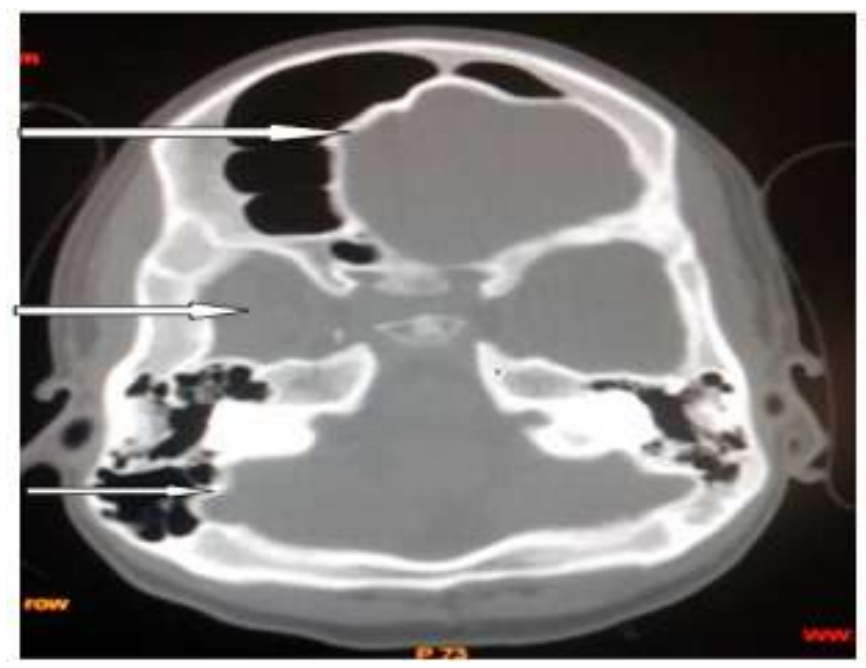

Figure 5: Axial brain CT bone window showing enlarged right ethmoidal sinus and mastoid air cells (long arrows) and the thickening of the calvarium on the right side (short arrow).

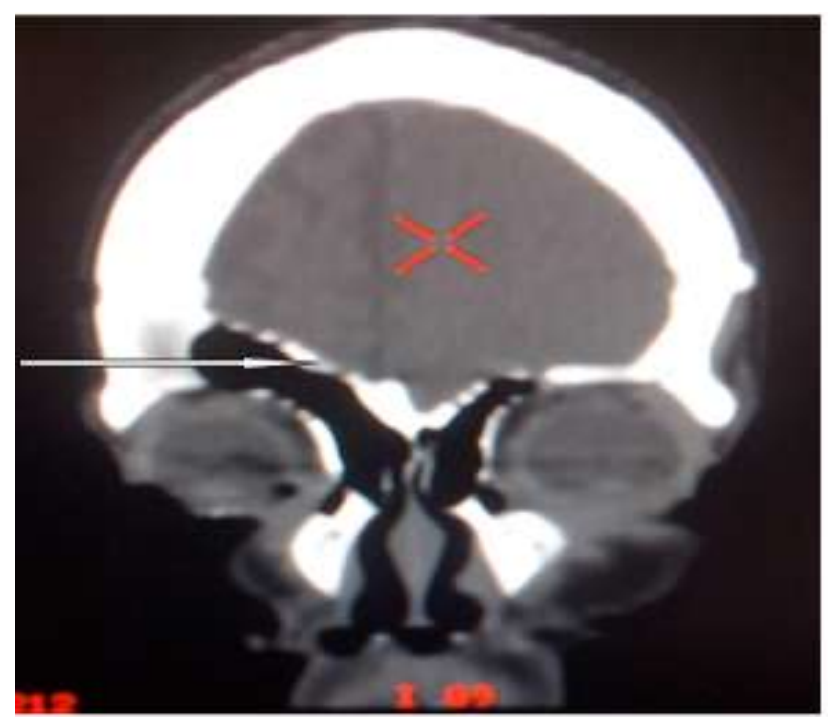

Figure 6: Coronal brain CT bone window showing enlarged right ethmoidal sinus (arrow). 


\section{Discussion}

Childhood cerebral hemiatrophy or Dyke-Davidoff-Masson syndrome (DDMS) is a rarely occurring condition that can be regarded as the end result of a number of different disease processes. It is characterized by marked asymmetry of the cerebral hemispheres $[1,2,5]$. This condition may be congenital or acquired. In the congenital or primary form, there are usually no evident aetiological factors and symptoms present at birth or shortly after. Cerebral damage is likely due to an intrauterine vascular occlusion during the prenatal period. The acquired form or secondary hemiatrophy often results from a cerebrovascular lesion, inflammatory process or cranial trauma in early childhood [3,6]. The index cases both had a history of febrile illness during early childhood and therefore insult to the brain due to inflammatory process inflicted by the febrile illness may be the possible aetiological factor.

The classical clinical presentation includes seizures which could be partial or generalized. Obajimi et al [7] in their study of computed tomography and childhood seizure disorder in Ibadan, reported a predominance of generalized tonic-clonic type of seizures in those with brain atrophy as reported in the index cases [7]. However, patients could also present with facial asymmetry, contra lateral hemiplegia or hemiparesis and mental retardation $[1,2,8]$. Seizures may appear months or years after the onset of hemiparesis [2]. The second case in addition had facial asymmetry with left sided hemiparesis.

Half and three fourth of adult brain sizes are attained during the first and third year of life respectively [9]. With brain growth, the brain presses outward on the calvarial tables resulting in the gradual enlargement and attainment of the general shape of the adult head. Identification of compensatory calvarial changes presuppose that cerebral abnormalities are the consequences of an atrophic or hypoplastic process from brain insults that started in early life postnatal life [9]. These skull changes correlate with the amount of underlying brain atrophy. These changes include thickening of the calvarium with loss of convolutional markings of the inner table and flattening of the calvarium. Hyper-pneumatization of paranasal sinuses and mastoid air cells lead to elevation of the petrous ridge [9]. These changes were demonstrated in the second case here presented (Figures 5 and 6).

Left preponderance of cerebral hemiatrophy has been reported [1,10]. Atalar $e$ t al detected left cerebral preponderance in 14 out of 19 cases studied [10]. This is in contrast with the cases in which both showed right sided cerebral hemiatrophy. However, hyperpneumatization as seen in the second patient was also seen in $26.11 \%$ of their cases. There has been reports of association of cerebral hemiatrophy $(\mathrm{CH})$ with other disorders [9,11]. Ozkan et al reported three cases of $\mathrm{CH}$ associated with haematological and developmental disorders. They reported association with thalassemia major in a 10 year old girl, acute myeloblastic leukemia and Marfan syndrome in a 23 year old man and craniosynostosis in a 1 year old boy [11]. Asli et al also reported a case of left $\mathrm{CH}$ with schizoaffective disorder [12]. No such associations were seen in cases here presented.

Computed tomography (CT) and magnetic resonance imaging (MRI) are the preferred modalities in assessment of the aetiology and lesion extent of cerebral parenchymal disease in atrophy, seizures, hemiparesis/plegia and craniofacial asymmetry [4,7]. CT has advantage of demonstrating the calvarial changes in addition to parenchymal changes (Figure 5). Imaging findings in CT include unilateral cerebral sulci prominence with dilatation of the ipsilateral lateral ventricle and Sylvian fissure, and the shift of the midline to the side of the atrophy [2] as demonstrated in both cases (Figures 1,2 and 4). Other changes are thickening of the ipsilateral frontal calvarium and squamous portion of the temporal bone, as well as enlargement of the ipsilateral frontal sinus and mastoid air cells $[2,13]$. These features were demonstrated in the second case presented.MRI is a valuable method of examination in the analysis of cerebral hemiatrophy as it can demonstrate changes in the cerebral hemispheres as well as highlighting bony structural changes and thus differentiating between congenital and acquired types of DDMS [4,7].

MRI was not done on these patients as they could not afford the costs. The differential diagnosis of DDMS include Stuge-Weber syndrome, progressive multi focal leucoencephalopathy and neurofibromatosis $[1,13]$. Stuge-Weber syndrome represents cerebral atrophy associated with leptomeningeal angioma. The patients have seizure disorders, mental retardation and hemiparesis. The distinguishing features are presence of port wine facial nevus, intracranial calcification and absence of ipsilateral midline and falx attachment shift. Midline shift is seen in late atrophic stage of progressive multifocal leucoencephalopathy but the absence of unilateral calvarial changes and decrease in brain size help to differentiate this condition [13]. Unilateral cerebral atrophy in neurofibromatosis is accompanied by compensatory hypertrophy of contralateral cerebrum. Hemimegalencephaly, gliosis cerebri, widespread cortical dysplasia are other features of neurofibromatosis [1]. The treatment of cerebral hemiatrophy is symptomatic, and should target convulsion, hemiplegia, hemiparesis and learning difficulties [2] as was done in these cases. Prognosis is better if hemiparesis occurs after the age of 2 years and in the absence of prolonged or recurrent seizures. Children with intractable disabling seizures and hemiplegia are potential candidates for hemispherectomy with a success rate of $85 \%$ in carefully selected cases [14]. 


\section{Conclusion}

We report two cases of childhood cerebral hemiatrophy (Dyke-Davidoff-Masson syndrome) with seizures and were diagnosed by computed tomography. The patients showed clinical improvement on medical treatment and are now on routine follow up.

\section{References}

[1]. F.U. Uduma, J.C. Emejulu, M. Motah, P.C. Okere, P.C. Ongolo and W. Muna. Differential diagnosis of cerebral hemiatrophy in childhood: A review of literature with illustrative report of two cases. Global J Health Sci., 5(3), 2013, 195-207.

[2]. J.B. Ndahiriwe, E. Cook . Dyke-Davidoff-Masson syndrome: A rare imaging case. Rwand Med J., 69(1), $2012,54-56$.

[3]. M.A. Duncan, S. Vazquez-Flores, E.B. Chaves-Lluevanos, A.C. Cantu-Salinas, L. deLeon-Flores, H.J. Villarreal-Velazquez. DykeDavidoff-Masson syndrome: A case study. Medicina universitaria, 16(63), 2014, 71-73.

[4]. V.K. Bakshi, P. Yadav, J. Chowdhary, H. Mishra and M. Shivnani. Cerebral hemiatrophy-role of CT and MRI in etiological stratification. 2015 www.posterng.netkey.at/.../index.php (accessed online on 17th December, 2015)

[5]. D. Negron, M.S. Correa-Rivas and L.E. Colon-Castillo. Cerebral hemiatrophy: An associated finding in an epileptic child. Puerto Rico Health Sci J. 22(2), 2003, 191-93.

[6]. J. Anupam, K.G. Rvindra, S.M. Hardeep, V. Rajesh, K.S. and Maneesh. Seizures in patients with cerebral hemiatrophy: A prognostic evaluation. Ann Indian Acad Neurol., 18, 2015, 39-44.

[7]. M.O. Obajimi, O.J. Fatunde, A.O. Ogunseyinde, O.O. Omigbodun, O.M. Atalabi and R.U. Joel. Computed tomography and childhood seizure disorder in Ibadan. West Afr J Med., 23(2), 2004, 167-172.

[8]. B. Shrestha. Acquired cerebral hemiatrophy: Dyke-Davidoff-Masson syndrome - A case report. Turk Neurosurg., 23(1), 2013, 117121.

[9]. S. Sharma, D. Goyal, A. Negi, R.G. Sood, A. Jhobta and M. Surya. Dyke Davidoff-Masson syndrome. Ind. J.Radiol. Imaging, 16(2), 2006, 165-166.

[10]. M.H. Ataler, D. Kagasioglu and F. Tas. Cerebral hemiatrophy (Dyke Davidoff-Masson syndrome) in childhood: Clinicoradiological analysis of 19 cases. Paed Intern. 49(1), 2007, 70-75.

[11]. O. Unal, H. Caksen, N. Kiymaz, I. Dilek, M. Kayan and O. Anlar. Cerebral hemiatrophy associated with haematological and developmental disorders. J Paed neurol. 2(3), 2004,169-172.

[12]. A.D. Tatlidede, A.D. Yalcin and T.G. Canpolat. Neurodevelopmental influences in psychosis: A case of left cerebral hemiatrophy and schizoaffective disorder. Clin Psychopharm., 23(4), 2013, 368-372.

[13]. L. Satija, S. Hariqbal, S.K. Khanna, S. Amarjit and A.P. Singh. Primary cerebral hemiatrophy. Med J Armed Forces India. 56, 2000, 59-60.

[14]. N.P. Narain, R. Kumar and B. Narain. Dyke Davidoff-Masson syndrome. Indian paed., 45, 2008, $927-928$. 